\title{
The pathophysiology of chronic noncommunicating hydrocephalus: lessons from continuous intracranial pressure monitoring and ventricular infusion testing
}

\author{
Per Kristian Eide, MD, PhD \\ Department of Neurosurgery, Oslo University Hospital-Rikshospitalet, Faculty of Medicine, University of Oslo, Norway
}

OBJECTIVE The pathophysiology of chronic noncommunicating hydrocephalus $(\mathrm{ncHC})$ is poorly understood. This present study explored whether lessons about the pathophysiology of this clinical entity might be retrieved from results of overnight monitoring of pulsatile and static intracranial pressure (ICP) and ventricular infusion testing.

METHODS The study cohort included adult patients (> 20 years of age) with chronic $\mathrm{ncHC}$ due to aqueductal stenosis in whom symptoms had lasted a minimum of 6 months. A reference cohort consisted of age- and sex-matched patients managed for communicating $\mathrm{HC}(\mathrm{cHC})$. Information about symptoms and clinical improvement following surgery was retrieved from a quality register, and results of overnight ICP recordings and ventricular infusion testing were retrieved from the hospital ICP database.

RESULTS The cohort with ncHC consisted of 61 patients of whom $6(10 \%)$ were managed conservatively, $34(56 \%)$ by endoscopic third ventriculostomy (ETV), and 21 (34\%) using ETV and subsequent shunt surgery. In patients responding to surgery, pulsatile ICP (mean ICP wave amplitude) was significantly increased to a similar magnitude in patients with $\mathrm{ncHC}$ and the reference cohort (cHC). Furthermore, intracranial compliance (ICC) was reduced in clinical responders. The results of ventricular infusion testing provided evidence that patients responding to ETV have impaired ventricular CSF absorption, while those requiring shunt placement after ETV present with impaired CSF absorption both in the intraventricular and extraventricular compartments.

CONCLUSIONS The study may provide some lessons about the pathophysiology of chronic ncHC. First, increased pulsatile ICP and impaired ICC characterize patients with chronic ncHC who respond clinically to CSF diversion surgery, even though static ICP is not increased. Second, in patients responding clinically to ETV, impaired ventricular CSF absorption may be a key factor. Patients requiring shunt placement for clinical response appear to have both intraventricular and extraventricular CSF absorption failure. A subgroup of patients with $\mathrm{ncHC}$ due to aqueductal stenosis has normal ventricular CSF absorption and normal ICC and may not be in need of surgical CSF diversion.

https://thejns.org/doi/abs/10.3171/2017.1.JNS162813

KEY WORDS noncommunicating hydrocephalus; communicating hydrocephalus; pathophysiology; ICP monitoring; ventricular infusion test

$\mathrm{H}$ YDROCEPHALUS (HC) is classified into 2 major subtypes, whether the obstruction of CSF flow is within CSF pathways of the cerebral ventricular system (noncommunicating $\mathrm{HC}[\mathrm{ncHC}]$ ) or the obstruction is over the brain surface (communicating $\mathrm{HC}[\mathrm{cHC}]$ ). This classification of HC was described by Dandy and Blackfan about a century ago ${ }^{5}$ and remains the basis for current classifications of $\mathrm{HC}^{.35,39}$
While there is consensus that acute obstruction of CSF pathways may cause acute and life-threatening $\mathrm{HC}$, the pathophysiology of chronic ncHC remains more controversial. $^{24}$ The traditional view is that obstruction of CSF flow results in increased static intracranial pressure (ICP), which in turn causes the cerebral ventricles to grow in size and secondarily affects the energy supply to the brain. ${ }^{24}$ Several subtypes of ncHC have been described that con-

ABBREVIATIONS $\mathrm{CHC}=$ communicating $\mathrm{HC} ; \mathrm{dP}=$ single wave pressure amplitude; $\mathrm{dT}=$ single wave rise time; $\mathrm{ETV}=$ endoscopic third ventriculostomy; EVD = external ventricular drain; $\mathrm{HC}=$ hydrocephalus; ICC = intracranial compliance; ICE = intracranial elastance; ICP = intracranial pressure; IIH = idiopathic intracranial hypertension; $\mathrm{iNPH}=$ idiopathic normal pressure hydrocephalus; ISF = interstitial fluid; LIAS = late-onset idiopathic aqueduct stenosis; LOVA = long-standing overt ventriculomegaly in adults; MWA = mean ICP wave amplitude; $\mathrm{ncHC}=$ noncommunicating $\mathrm{HC}$; $\mathrm{R}_{\text {out }}=$ resistance to CSF outflow.

SUBMITTED November 8, 2016. ACCEPTED January 26, 2017.

INCLUDE WHEN CITING Published online August 11, 2017; DOI: 10.3171/2017.1.JNS162813. 
sider the clinical and radiological presentations and the duration of disease development. ${ }^{21,36}$

However, several clinical observations regarding chronic ncHC may be difficult to explain based on the current knowledge. Endoscopic third ventriculostomy (ETV) is the preferred treatment of $\mathrm{ncHC}$, aiming at establishing an alternative route for CSF flow. However, while ETV may be very successful in many individuals, ${ }^{28,48}$ the success rate (i.e., need for a shunt after ETV) varies substantially between studies, particularly depending on age and duration of disease. ${ }^{44}$ Adults with chronic ncHC represent a particular challenge, of whom a substantial number of individuals may require shunting after ETV. Hence, in one study the failure rate of ETV was as high as $50 \% .{ }^{45} \mathrm{~A}$ recent systematic review and meta-analysis concluded that there is insufficient proof to unequivocally recommend one mode of treatment over the other in ncHC..$^{38}$ Another puzzling observation is that in some patients treated with ETV for ncHC, the static ICP (mean ICP) may remain increased. ${ }^{3,37}$ Adding to these observations, we found that neither the static nor the pulsatile ICP associated with the ventricule size in chronic ncHC. ${ }^{41}$ Moreover, it is a clinical experience that a few individuals with chronic ncHC due to aqueductal stenosis manage well without any surgery.

To improve the management of chronic ncHC in adults, we need to better understand the pathophysiology behind this condition. The present study was undertaken to explore results of continuous overnight measurements of static and pulsatile ICP and ventricular infusion testing. Adult patients with chronic ncHC were compared with a reference group, consisting of age/gender-matched individuals managed for chronic $\mathrm{cHC}$.

\section{Methods}

\section{Patients and Study Design}

The study was approved by the Oslo University Hospital-Rikshospitalet as a quality study. The Regional Committee for Medical and Health Research Ethics of Health Region South-East, Norway, was informed in writing and had no objections to the study. Both the study cohort (ncHC cases) and the reference subjects (individuals with cHC) were retrieved from a Neurovascular-Hydrocephalus Quality Register, including consecutive patients managed within the Department of Neurosurgery, Oslo University Hospital-Rikshospitalet, during the years 2002-2012.

Criteria for inclusion in the study cohort, denoted ncHC, were as follows: 1) age $>20$ years; 2 ) an MRI-verified diagnosis of ncHC due to stenosis of the aqueduct of Sylvius; 3 ) presence of symptoms that had been lasting $>6$ months; and 4) performance of overnight ICP monitoring and/or ventricular infusion testing as part of preoperative assessment, with storage of the continuous pressure signals as raw data files.

Exclusion criteria were as follows: 1) ncHC due to an expansive process such as tumor (except of tectum hyperplasia), cyst, or vascular malformation, causing aqueductal stenosis; and 2) previous surgical treatment for HC.

Criteria for inclusion as a reference subject, denoted as $\mathrm{cHC}$, were as follows: 1) each reference subject was matched with a study participant according to age and sex, and also according to management; 2) each reference patient had undergone similar management, either conservative or surgical (i.e., shunt surgery) and had been categorized as either a clinical nonresponder or clinical responder similar to the individuals of the study cohort; 3 ) an MRI-verified diagnosis of cHC, i.e., no evidence of stenosis of the aqueduct of Sylvius; 4) presence of symptoms that had been lasting > 6 months; and 5) performance of overnight ICP monitoring and/or ventricular infusion testing as part of preoperative assessment, with storage of the continuous pressure signals as raw data files. To avoid bias, reference subjects were retrieved from the quality register according to the above-mentioned criteria before pressure data were retrieved from the ICP database.

\section{Clinical Assessment, Indication for Surgery, and Outcome}

The Neurovascular-Hydrocephalus Quality Registry stores clinical information about preoperative symptoms, imaging findings, and follow-up data. The information of the quality register had been obtained as part of patient management. Patients managed surgically are categorized as clinical responders or nonresponders. Patients were examined clinically at the outpatient clinic $6-12$ months after surgery; responders had improvement of symptoms, while nonresponders reported no clinical improvement of the symptoms specified in Table 1.

The department's routine management is briefly described. Patients with ncHC are typically referred from local neurological departments, based on imaging findings of ventriculomegaly and obstruction of CSF pathways. Within the department, patient management consists of clinical neurological assessment, imaging (MRI), and invasive pressure measurement, either overnight continuous ICP monitoring or ventricular infusion testing. The indication for surgery is based on the combination of clinical findings, presence of comorbidity, imaging findings, and results of ICP monitoring. First-order surgical treatment of $\mathrm{ncHC}$ is ETV, while second-order treatment is shunt surgery (ventriculoperitoneal shunt).

With regard to ETV, the department's routine is as follows: ETV is performed with the patient under general anesthesia, with the patient's head fixed in a 3-point head holder. After making a bur hole at the coronal suture, close to the midline, a rigid endoscope is passed into the lateral ventricle and the third ventricle via the foramen of Monroe. A deflated balloon catheter is used to penetrate the floor of the third ventricle between the infundibula recess of the pituitary stalk and the anterior border of the mammillary bodies. The fenestration is expanded to about 5 $\mathrm{mm}$ using the rigid endoscope. Prepontine arachnoid adhesions are routinely opened using the balloon catheter.

With regard to shunt surgery, the department's routine is to place a ventriculoperitoneal shunt, most commonly a Codman Hakim programmable valve (Codman, Johnson \& Johnson), using an opening pressure of $12 \mathrm{~cm} \mathrm{H}_{2} \mathrm{O}$.

Patients managed conservatively or surgically are followed in the outpatient clinic at regular intervals, focusing on clinical development and imaging of ventriculomegaly. The surgeon performing the ETV and/or shunt procedure follows his or her patients at the outpatient clinic. 
TABLE 1. Demographic, clinical, and radiological data of ncHC and $\mathrm{cHC}$ cohorts

\begin{tabular}{|c|c|c|c|}
\hline Variable & ncHC Cohort & $\mathrm{cHC}$ Cohort & p Value* \\
\hline \multicolumn{4}{|l|}{ Demographic data } \\
\hline No. of patients & 61 & 61 & \\
\hline Mean age \pm SD (yrs) & $52.8 \pm 14.1$ & $57.6 \pm 11.4$ & 0.04 \\
\hline $\operatorname{Sex}(F / M)$ & $27 / 34$ & $27 / 34$ & \\
\hline \multicolumn{4}{|l|}{ Clinical symptoms/findings (\%) } \\
\hline Gait disturbance & $44(72)$ & $55(90)$ & 0.01 \\
\hline Incontinence & $22(36)$ & $42(69)$ & $<0.001$ \\
\hline Cognitive impairment & $39(64)$ & $54(89)$ & 0.001 \\
\hline Headache & $30(49)$ & $6(10)$ & $<0.001$ \\
\hline Nausea & $4(7)$ & $2(3)$ & NS \\
\hline Dizziness & $21(34)$ & $3(5)$ & $<0.001$ \\
\hline Lethargy & $5(8)$ & $5(8)$ & NS \\
\hline Visual disturbances & $5(8)$ & $4(7)$ & NS \\
\hline Mean duration of symptoms in mos \pm SD & $27.6 \pm 3.5$ & $43.2 \pm 42.3$ & 0.02 \\
\hline \multicolumn{4}{|l|}{ Ventricular volume (mean \pm SD) } \\
\hline Evan's index & $0.44 \pm 0.09$ & $0.41 \pm 0.12$ & NS \\
\hline Ventricular score & $113.9 \pm 23.4$ & $106.0 \pm 31.2$ & NS \\
\hline Ventricular volume in $\mathrm{ml}$ & $289.8 \pm 241.9$ & $250.4 \pm 275.1$ & NS \\
\hline ICV in $\mathrm{ml}$ & $1512.5 \pm 331.4$ & $1578.7 \pm 342.5$ & NS \\
\hline Ventricular volume index as $\%$ of ICV & $17.8 \pm 10.1$ & $14.3 \pm 12.6$ & NS \\
\hline \multicolumn{4}{|l|}{ Invasive diagnostic procedures (\%) } \\
\hline Overnight ICP monitoring & $53(87)$ & $61(100)$ & \\
\hline Ventricular infusion testing & $45(74)$ & $15(25)$ & \\
\hline \multicolumn{4}{|l|}{ Treatment (\%) } \\
\hline Conservative & $6(10)$ & $6(10)$ & \\
\hline ETV & $34(56)$ & - & \\
\hline ETV followed by shunt placement & $21(34)$ & - & \\
\hline Shunt & - & $55(90)$ & \\
\hline \multicolumn{4}{|l|}{ Outcome after surgery (\%) } \\
\hline Responders & $41(74.5)$ & $41(74.5)$ & \\
\hline Nonresponders & $14(25.5)$ & $14(25.5)$ & \\
\hline
\end{tabular}

\section{Radiological Assessment of Aqueductal Stenosis and Ventricular Volume}

The diagnosis of ncHC was based on MRI acquisitions, either from the referring hospitals or obtained in our hospital, provided that additional MRI sequences were required. All MRI scans were reassessed by a neuroradiologist at our hospital. This hospital's routine for diagnosis of ncHC is: 1) Siemens 1.5-T MRI, consisting of T1-weighted, T2-weighted, and CISS (Siemens-specific abbreviation for gradient echo steady-state sequence), transversal T2-weighted and diffusion, and coronal FLAIR; and 2) Siemens 3-T MRI, consisting of sagittal T1-weighted 3D MPRAGE, sagittal T2-weighted space, sagittal FLAIR-volume, transversal T2-weighted, and diffusion.

With regard to ventricular volume $\left(\mathrm{cm}^{3}\right)$ estimation, calculations were performed using the integrated $3 \mathrm{D}$ volume functions in iPlan software (BrainLAB), including the 3D space of the lateral ventricles, third ventricle, aqueduct, fourth ventricle, and total intracranial volumes of both the supra- and infratentorial compartments. The ventricular volume index was calculated as a percentage of ventricular volume of total intracranial volume.

\section{Preoperative ICP Monitoring and Ventricular Infusion Testing}

During the study period, we have used diagnostic overnight ICP monitoring (since 2002) and/or ventricular infusion testing (since 2007) on a routine basis to aid in the management of chronic ncHC; since 2007, we aimed to use both tests.

For monitoring ICP, a bur hole was made in the skull under local or general anesthesia. A Codman ICP microsensor (Johnson \& Johnson) was zeroed against the atmo- 
spheric pressure and placed $1-2 \mathrm{~cm}$ into the brain parenchyma via a minor opening in the dura. After placement of the ICP sensor, the patient was returned to the ward and the ICP sensor became connected to a computerized system for continuous recording. The monitoring is continued overnight, with real-time presentation of the continuous ICP signals.

Patients undergoing a ventricular infusion test received an external ventricular drain (EVD) with a built-in Codman ICP MicroSensor (Codman external drainage with ICP sensor; Codman/Johnson \& Johnson), introduced into the frontal horn of the cerebral ventricles. After placement of the EVD with an ICP sensor, the patient returned to the ward keeping the EVD closed, and the ICP monitoring started and continued until the next day, using the computerized system described above. Ventricular infusion testing was performed with the patient awake. The ventricular infusion tests consisted of 3 phases, as previously described..$^{10} \mathrm{ICP}$ was first recorded for a few minutes (opening phase). Then, Ringer solution was infused via the EVD at a standard infusion rate of $1.5 \mathrm{ml} / \mathrm{min}$ until a plateau pressure was reached (plateau phase). The entire period of infusion was denoted the infusion phase. If ICP increased markedly without reaching a plateau, the infusion was ended.

\section{Analysis of Continuous Pressure Signals}

The continuous ICP waveforms of overnight monitoring and infusion testing were sampled at 100-200 Hz. The continuous pressure signals were retrieved from the ICP database, and analyzed according to a previously published method for automatic analysis of ICP waves (Sensometrics Software, dPCom As). ${ }^{11}$ In brief, the cardiac-induced waves are automatically identified by their beginning and ending diastolic minimum pressures and the systolic maximum pressures. Only 6-second time windows containing a minimum of 4 cardiac beat-induced waves were used for the present analysis. Then, the amplitude ("pulse amplitude," dP), rise time (dT), and rise time coefficient $(\mathrm{dP} / \mathrm{dT})$ are determined from the identified cardiac beat-induced ICP waves. For each 6-second time window, the output of pulsatile ICP was the mean ICP wave amplitude (MWA). The output of static ICP (each 6-second time window) was the mean ICP, which was determined independent of the cardiac beat-induced waves.

Average values of MWA and mean ICP were determined for a standardized recording time from $11 \mathrm{PM}$ to 7 AM. In addition, the percentage of time with MWA and mean ICP above defined thresholds was determined. Based on previous results, ${ }^{18}$ we recommend surgery in patients with MWA values above upper normal threshold values during the night period, i.e., MWA $\geq 4 \mathrm{~mm} \mathrm{Hg}$ on average and $\geq 5 \mathrm{~mm} \mathrm{Hg}$ in a minimum $10 \%$ of recording time from 11 PM until 7 AM. Trend plots of MWA and mean ICP from 11 PM to $7 \mathrm{AM}$, and also one 6-second time window with the cardiac beat-induced ICP waves, from 1 patient are presented in Fig. 1.

With regard to the ventricular infusion tests, the MWA and mean ICP scores were determined for the different phases (opening, plateau, and infusion phases). In addi- tion, the software automatically determines the intracranial compliance (ICC) during infusion, as previously described. ${ }^{11}$ The method for determining ICC is as follows: the pressure difference (D) is computed over 15-second time windows as the difference in mean ICP determined over 3-second periods at the end $\left(\mathrm{P}_{\mathrm{n}}\right)$ and beginning $\left(\mathrm{P}_{\mathrm{n}-}\right.$ $\left.{ }_{1}\right)$ of the 15-second time window $\left(D=P_{n}-P_{n-1}\right)$. Hence, while $P_{n-1}$ represents time $0-3$ seconds, $P_{n}$ represents time $12-15$ seconds. During this 15 -second period, the volume change is $0.375 \mathrm{ml}$ (corresponding to an infusion rate of $1.5 \mathrm{ml} / \mathrm{min})$. Accordingly, ICC $=0.375 \mathrm{ml} /\left(\mathrm{P}_{\mathrm{n}}-\mathrm{P}_{\mathrm{n}-1}\right)$. The software applies a moving time window, allowing an updated value every subsequent 3 -second period. For each infusion test, the ICC represents the average of all the 3 -second periods during the infusion period. Moreover, during infusion testing, the resistance to CSF outflow $\left(R_{\text {out }}\right)$ was calculated as the difference between the plateau pressure $\left(\mathrm{P}_{\mathrm{p}}\right)$ and the opening pressure $\left(\mathrm{P}_{\mathrm{o}}\right)$, divided by infusion rate $\left[\left(\mathrm{P}_{\mathrm{p}}-\mathrm{P}_{\mathrm{o}}\right) / 1.5 \mathrm{ml} / \mathrm{min}\right] . .^{13}$

\section{Statistical Analysis}

SPSS statistical software (version 22, IBM Corp.) was used for statistical analysis. Differences between groups were determined using 1-way ANOVA, and Bonferronicorrected post hoc tests when comparing more than 2 groups. Significant differences between categorical data were determined using the Pearson chi-square test. Associations between observations were determined by the Pearson correlation coefficient. Significance was accepted at the 0.05 level.

\section{Results \\ Study Cohort}

The study cohort included 61 patients with chronic ncHC due to aqueductal stenosis managed within our department during the 11-year period from 2002 to 2012 (Table 1). All patients had undergone overnight ICP monitoring and/or ventricular infusion testing. Likewise, the cHC (reference) cohort consisted of 61 age- and sexmatched individuals who also had undergone overnight ICP monitoring and/or ventricular infusion testing. Table 1 shows the demographic data of these 2 cohorts. Despite the best possible age match, the $\mathrm{cHC}$ cohort was significantly older than the ncHC cases (mean 57.6 vs 52.8 years old, respectively).

The symptom profile differed somewhat between the study and reference cohorts. While headache and dizziness were significantly more prevalent in the ncHC patients, gait disturbance, incontinence, and cognitive impairment occurred more frequently in the $\mathrm{cHC}$ group (Table 1).

There were neither any significant differences in CTbased linear ventricular size measures, nor in MRI-based ventricular volumes between the 2 patient cohorts (Table 1). In all study patients ( $\mathrm{ncHC}$ ), MRI verified the presence of aqueductal stenosis, as detailed in the Methods section. Figure 2 shows the aqueductal stenosis in 3 individuals for each of the management cohorts: conservative management, surgery group (nonresponders), and surgery group (responders). 
A

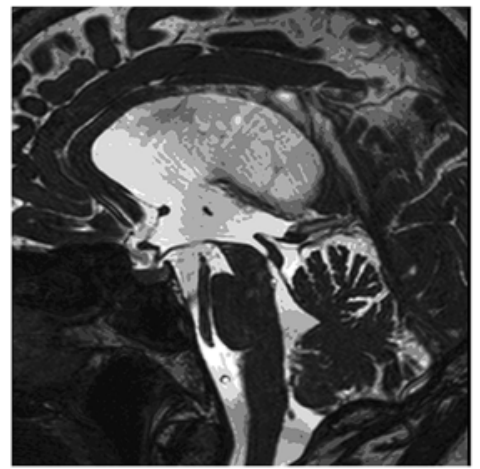

B
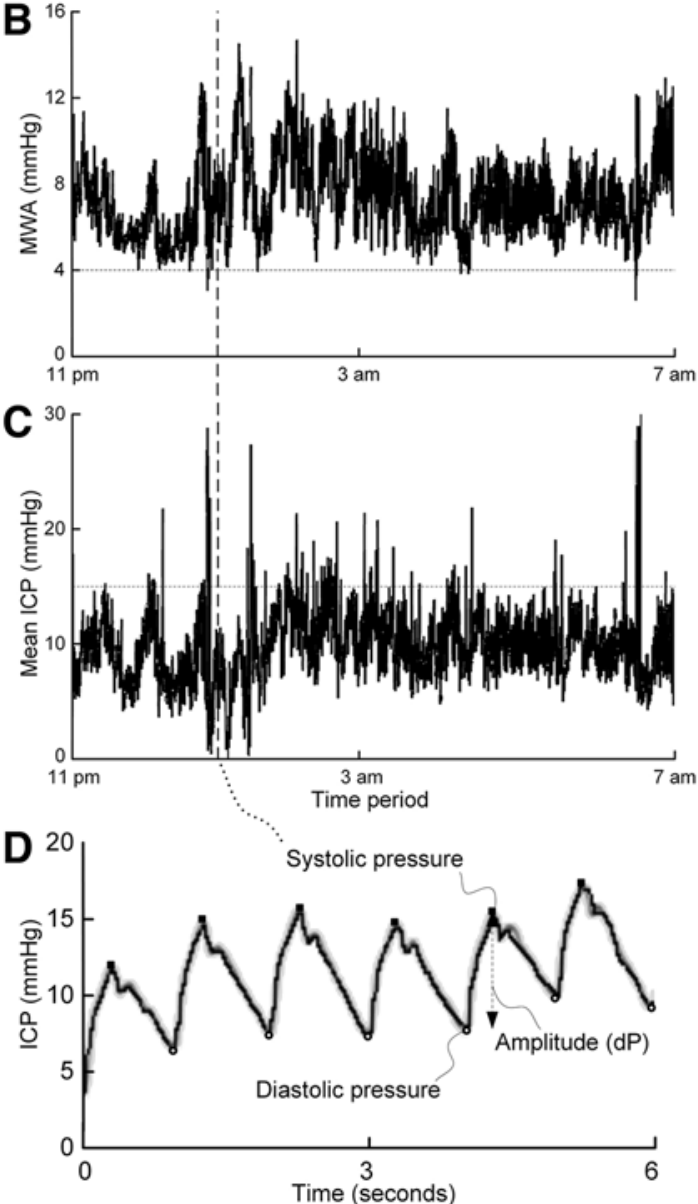

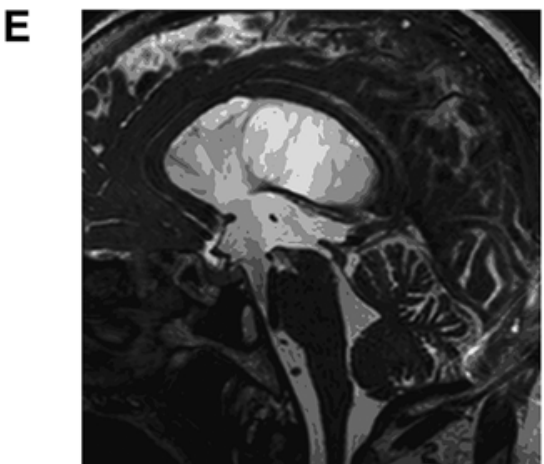

$\mathbf{F}$
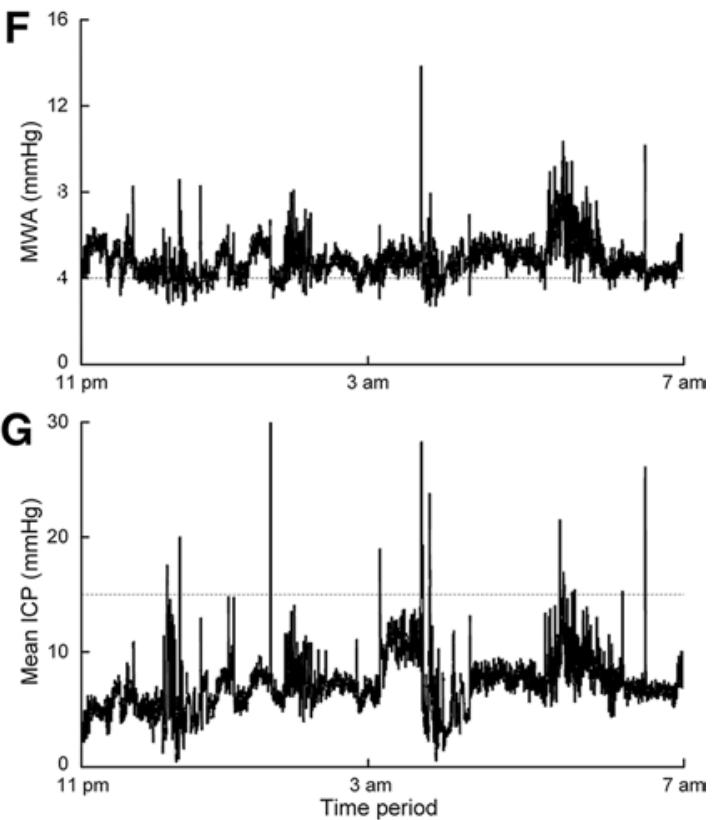

FIG. 1. MR images and overnight ICP scores before (A-C) and after (E-G) ETV in a 63-year-old woman with ncHC due to aqueductal stenosis are shown. Her sagittal cerebral MRI before ETV (A) reveals the aqueductal stenosis. The trend plots of MWA (B) and mean ICP (C) present consecutive values of MWA and mean ICP, respectively, computed every 6 seconds, from 11 PM to 7 AM. One 6-second time window is presented (D), with the individual cardiac beat-induced single ICP waves. The amplitude (dP) is the pressure difference between the systolic and diastolic pressures of the individual ICP waves. Her MRI obtained after ETV and before shunt surgery $(E)$ reveals an open stoma. After ETV, the trend plots from 11 PM to 7 AM of MWA (F) and mean ICP $(G)$ show that both MWA and mean ICP have become reduced. Hence, before ETV, the average MWA was $7.3 \mathrm{~mm} \mathrm{Hg}$ (panel B; MWA $\geq 5 \mathrm{~mm} \mathrm{Hg}$ in $93 \%$ and $\geq 6 \mathrm{~mm} \mathrm{Hg}$ in $74 \%$ of recording time from $11 \mathrm{PM}$ to $7 \mathrm{AM}$ ), while after ETV (and before shunting) average MWA was $4.8 \mathrm{~mm} \mathrm{Hg}$ (panel F; MWA $\geq 5 \mathrm{~mm} \mathrm{Hg}$ in $33 \%$ and $\geq 6 \mathrm{~mm} \mathrm{Hg}$ in $8 \%$ of recording time). The average of mean ICP was $9.9 \mathrm{~mm} \mathrm{Hg}$ before ETV (panel C; mean ICP $\geq 15 \mathrm{~mm} \mathrm{Hg}$ in $0 \%$ of recording time), whereas it was $7.1 \mathrm{~mm} \mathrm{Hg}$ after ETV (panel G; mean ICP $\geq 15 \mathrm{~mm} \mathrm{Hg}$ in $0 \%$ of recording time). This patient experienced her clinical improvement after shunt surgery despite a previously successful ETV.

Among the 61 patients with ncHC due to aqueductal stenosis, $6(10 \%)$ were managed conservatively without surgery, while $34(56 \%)$ underwent ETV, and 21 (34\%) ETV followed by shunt placement due to a lack of ex- pected clinical improvement (Table 1). Among those undergoing surgery, $41(74.5 \%)$ were categorized as clinical responders and $14(25.5 \%)$ as clinical nonresponders (11 in the ETV group and 3 in the ETV+shunt group). The 

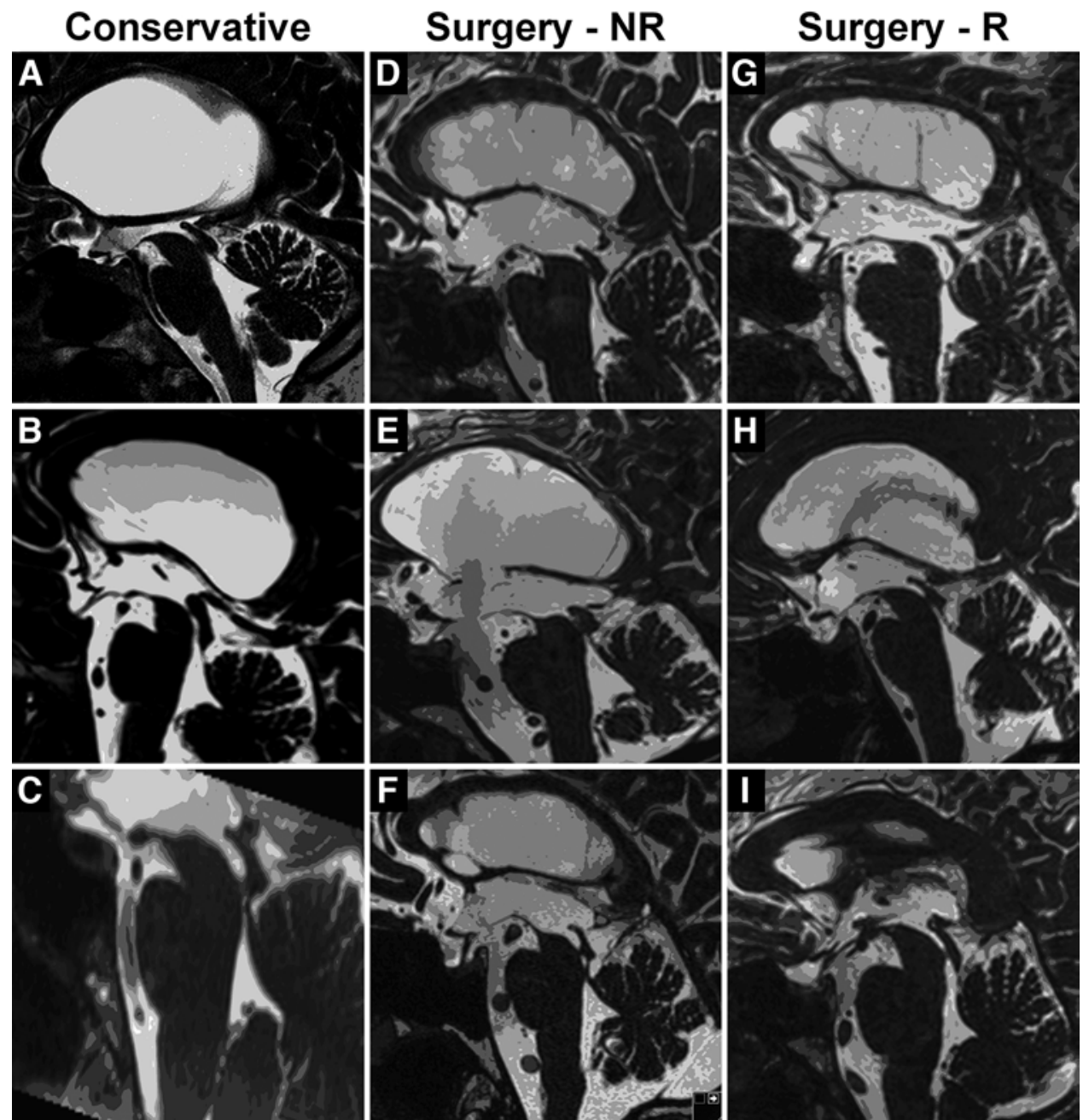

FIG. 2. The presence of $\mathrm{ncHC}$ due to aqueductal stenosis is illustrated in 9 different patients, including sagittal MR images from 3 patients in the conservative management group (a 27-year-old woman [A], a 55-year-old woman [B], and a 63-year-old woman [C]), 3 patients in the surgery (ETV+shunt) nonresponder (NR) group (a 69-year-old woman [D], a 63-year-old man [E], and a 67 -year-old man [F]), and 3 patients in the surgery responder (R) group (a 65-year-old man [G], a 48-year-old woman [H], and a 39-year-old man [I]).

distribution of responders/nonresponders was identical for the $\mathrm{cHC}$ cohort.

\section{Overnight ICP Scores and Ventricular Infusion Tests in ncHC and cHC Cohorts}

Complications of overnight ICP monitoring or ventricular infusion testing were not observed in this cohort. At the group level, there were no differences between the $\mathrm{ncHC}$ and $\mathrm{cHC}$ cohorts with regard to pulsatile or static ICP scores during overnight monitoring (Table 2). Similarly, the results of ventricular infusion testing were comparable between both patient cohorts, although pressure scores tended to be more abnormal in the $\mathrm{cHC}$ cohort (MWA during infusion was close to significantly higher in the cHC cohort). Taken together, neither the pulsatile nor the static ICP scores were more abnormal in ncHC patients.

Table 3 presents the results of overnight ICP monitoring and ventricular infusion testing in $\mathrm{ncHC}$ and $\mathrm{cHC}$ cohorts as categorized according to management (conservative/ surgery) and clinical outcome (nonresponder/responder). The main observation was that pulsatile ICP (MWA) was comparably elevated in $\mathrm{ncHC}$ and $\mathrm{cHC}$ patients responding to surgery, as compared with patients not responding to surgery, or patients managed conservatively. This aspect is further illustrated in Fig. 3. The results of overnight ICP monitoring revealed increased average MWA (Fig. 3A), as well as increased percentage of MWA $>5 \mathrm{~mm} \mathrm{Hg}$ (Fig. 3B) or $>6 \mathrm{~mm} \mathrm{Hg}$ (Fig. 3C) in surgical responders, while mean ICP was not significantly different between the management and outcome groups (Fig. 3D-F).

With regard to the results of ventricular infusion testing, pulsatile ICP (MWA) was increased and ICC impaired in those responding to surgery (Table 3 ). It should be noted that ncHC and $\mathrm{cHC}$ patients responding to surgery showed comparable results.

\section{ICP Scores and Ventricular Infusion Test Results in ncHC According to Surgical Modality}

The overnight ICP scores and results of ventricular 
infusion testing in $\mathrm{ncHC}$ patients according to surgical modality (ETV/ETV+shunt) and outcome (nonresponder/ responder) are presented in Table 4. As compared with nonresponders, the responders had significantly higher MWA values both during overnight monitoring and during infusion testing (plateau and infusion phases), and significantly lower ICC (ETV subcohort, Table 4). No significant differences in MWA were found between responders who had undergone ETV or ETV+shunt. Figure 4 further illustrates the elevated MWA during overnight monitoring in responders (Fig. 4A-C), while no significant differences were found in mean ICP (Fig. 4D-F). Moreover, Fig. 5 illustrates that in responders, during infusion the MWA was increased (Fig. 5A), mean ICP unchanged (Fig. 5B), and ICC reduced (Fig. 5C).

One of the patients in the ETV+shunt responder subgroup is illustrated in Fig. 1. This 63-year-old woman had ventriculomegaly and aqueductal stenosis (Fig. 1A). Overnight ICP monitoring revealed raised pulsatile ICP (MWA, Fig. 1B), although modestly increased static ICP (mean ICP, Fig. 1C). After ETV, MRI revealed an open stoma (Fig. 1E), but she had lasting symptoms (unsteady gait, cognitive impairment, headache, and blurred vision). A new overnight ICP monitoring was performed, which showed lasting elevated pulsatile ICP (Fig. 1F). The patient then received a ventriculoperitoneal shunt and thereafter was relieved of her symptoms.

\section{Pulsatile ICP and ICC}

The association between pulsatile ICP (MWA) and ICC was examined in patients with ncHC (Fig. 6). The ICC measured during ventricular infusion correlated significantly with MWA recorded overnight (Fig. 6A) and MWA measured during the opening phase (Fig. 6C), plateau phase (Fig. 6E), and infusion phase (Fig. 6G). In contrast, no significant association between ICC and mean ICP was found (Fig. 6B, D, F, and H).

\section{Discussion}

In treatment-responsive patients with ncHC due to aqueductal stenosis, overnight ICP monitoring provided evidence of increased pulsatile ICP and impaired ICC, despite normal static ICP. Findings were similar in patients with $\mathrm{ncHC}$ and $\mathrm{cHC}$. Ventricular infusion testing provided evidence of variable degrees of ventricular CSF absorption in $\mathrm{ncHC}$, which determines the success rate of ETV. The individuals managing well without surgery have intact ventricular CSF absorption and normal ICC.

\section{Acute Versus Chronic ncHC and the Classification of $\mathrm{HC}$}

ncHC may develop acutely because of tumors, bleeds, infections, and other causes. It may be life threatening and require immediate intervention. In a historical perspective, management of ncHC was a major challenge, with high mortality rates. ${ }^{14} \mathrm{CSF}$ diversion by ETV or shunting can be lifesaving.

The present study cohort included patients with chronic ncHC in whom symptoms had lasted for several months. Accordingly, the present results apply to chronic and not acute ncHC.
TABLE 2. ICP/infusion test scores of ncHC patients compared with $\mathrm{CHC}$ patients

\begin{tabular}{|c|c|c|c|}
\hline Testing Variable & $\mathrm{ncHC}$ & $\mathrm{cHC}$ & $p$ Value \\
\hline \multicolumn{4}{|l|}{ Overnight ICP monitoring† } \\
\hline \multicolumn{4}{|l|}{ MWA } \\
\hline Average $(\mathrm{mmHg})$ & $4.6 \pm 1.8$ & $4.8 \pm 1.8$ & NS \\
\hline$\% \geq 5 \mathrm{mmHg}$ & $32 \pm 34$ & $36 \pm 33$ & NS \\
\hline$\% \geq 6 \mathrm{mmHg}$ & $21 \pm 29$ & $21 \pm 28$ & NS \\
\hline \multicolumn{4}{|l|}{ Mean ICP } \\
\hline Average $(\mathrm{mmHg})$ & $7.6 \pm 4.6$ & $8.3 \pm 3.7$ & NS \\
\hline$\% \geq 15 \mathrm{mmHg}$ & $8 \pm 14$ & $7 \pm 11$ & NS \\
\hline$\% \geq 20 \mathrm{mmHg}$ & $2 \pm 5$ & $1 \pm 2$ & NS \\
\hline \multicolumn{4}{|l|}{ Ventricular infusion testing } \\
\hline \multicolumn{4}{|l|}{ Opening phase } \\
\hline MWA (mmHg) & $3.0 \pm 1.4$ & $3.7 \pm 1.2$ & NS \\
\hline Mean ICP (mmHg) & $7.4 \pm 4.6$ & $7.7 \pm 3.4$ & NS \\
\hline \multicolumn{4}{|l|}{ Plateau phase } \\
\hline MWA (mmHg) & $7.4 \pm 3.6$ & $8.8 \pm 3.2$ & NS \\
\hline Mean ICP (mmHg) & $21.6 \pm 6.8$ & $22.1 \pm 7.3$ & NS \\
\hline \multicolumn{4}{|l|}{ Infusion phase } \\
\hline MWA (mmHg) & $5.1 \pm 2.2$ & $6.3 \pm 1.9$ & 0.05 \\
\hline Mean ICP $(\mathrm{mmHg})$ & $15.1 \pm 4.6$ & $15.3 \pm 4.5$ & NS \\
\hline $\mathrm{ICC}(\mathrm{ml} / \mathrm{mmHg})$ & $0.56 \pm 0.12$ & $0.55 \pm 0.21$ & NS \\
\hline $\mathrm{R}_{\text {out }}(\mathrm{mmHg} / \mathrm{ml} / \mathrm{min})$ & $9.5 \pm 4.1$ & $9.6 \pm 3.9$ & NS \\
\hline Infusion volume (ml) & $16.5 \pm 5.3$ & $16.7 \pm 6.3$ & NS \\
\hline \multicolumn{4}{|c|}{$\begin{array}{l}\text { Data presented as mean } \pm \text { SD. } \\
\text { * Significant differences between the } 2 \text { groups were determined by } 1 \text {-way } \\
\text { ANOVA. } \\
\dagger \text { Overnight ICP parameters were recorded from } 11 \text { PM to } 7 \mathrm{AM} \text {. }\end{array}$} \\
\hline
\end{tabular}

The current classifications of $\mathrm{HC}$ primarily consider the site of CSF obstruction. ${ }^{39}$ A classification based on a more comprehensive assessment of the various aspects of $\mathrm{HC}$ has also been proposed. ${ }^{35}$ With regard to chronic ncHC due to aqueductal stenosis, previously various notations have been used, primarily considering the clinical and radiological presentations and the duration of disease. It was recognized early that a "benign" form of chronic ncHC due to aqueductal stenosis could manifest in adulthood. ${ }^{26}$ Fukuhara and Luciano introduced the term "late-onset idiopathic aqueduct stenosis" (LIAS). ${ }^{21}$ Oi introduced the term "long-standing overt ventriculomegaly in adults" (LOVA) to describe a clinical entity in adults with chronic ncHC due to aqueductal stenosis, probably developing from childhood, and characterized by increased ICP, dementia, and/or impaired IQ. ${ }^{36}$ The author proposed ETV as preferred treatment. However, controversy still exists as to the classification and understanding of chronic adult ncHC. ${ }^{39}$

\section{Surgery for Chronic ncHC}

Based on the present management results, the individuals with ncHC due to aqueductal stenosis were divided into 3 main categories: 1) individuals not requiring surgical treatment (conservative group); 2) individuals in whom ETV was sufficient (ETV group); and 3) individu- 
TABLE 3. ICP/infusion test scores of $\mathrm{ncHC}$ and $\mathrm{cHC}$ patients according to management

\begin{tabular}{|c|c|c|c|c|c|c|}
\hline \multirow[b]{2}{*}{ Testing Variable } & \multicolumn{2}{|c|}{ Conservative } & \multicolumn{2}{|c|}{ Surgery (nonresponders) } & \multicolumn{2}{|c|}{ Surgery (responders) } \\
\hline & $\mathrm{ncHC}$ & $\mathrm{cHC}$ & $\mathrm{ncHC}$ & $\mathrm{cHC}$ & $\mathrm{ncHC}$ & $\mathrm{cHC}$ \\
\hline \multicolumn{7}{|l|}{ Overnight ICP monitoring* } \\
\hline \multicolumn{7}{|l|}{ MWA } \\
\hline Average $(\mathrm{mmHg})$ & $3.3 \pm 0.7 \ddagger$ & $3.3 \pm 0.4 \ddagger$ & $3.0 \pm 0.4 \S$ & $3.1 \pm 0.6 \S$ & $5.4 \pm 1.6$ & $5.5 \pm 1.7$ \\
\hline$\% \geq 5 \mathrm{mmHg}$ & $5 \pm 7 \ddagger$ & $5 \pm 6 \ddagger$ & $2 \pm 2 \S$ & $6 \pm 5 \S$ & $47 \pm 33$ & $51 \pm 31$ \\
\hline$\% \geq 6 \mathrm{mmHg}$ & $1 \pm 2$ & $1 \pm 2$ & $0 \pm 1 \ddagger$ & $2 \pm 2 \ddagger$ & $31 \pm 31$ & $31 \pm 29$ \\
\hline \multicolumn{7}{|l|}{ Mean ICP } \\
\hline Average $(\mathrm{mmHg})$ & $7.0 \pm 2.7$ & $7.4 \pm 5.4$ & $5.2 \pm 4.6$ & $7.6 \pm 3.6$ & $8.6 \pm 4.7$ & $8.7 \pm 3.5$ \\
\hline$\% \geq 15 \mathrm{mmHg}$ & $2 \pm 4$ & $5 \pm 6$ & $4 \pm 12$ & $3 \pm 4$ & $11 \pm 15$ & $9 \pm 12$ \\
\hline$\% \geq 20 \mathrm{mmHg}$ & $1 \pm 3$ & $1 \pm 1$ & $1 \pm 2$ & $1 \pm 1$ & $3 \pm 6$ & $1 \pm 2$ \\
\hline \multicolumn{7}{|l|}{ Ventricular infusion testing } \\
\hline \multicolumn{7}{|l|}{ Opening phase } \\
\hline MWA (mmHg) & $2.9 \pm 0.6$ & $2.7 \pm 0.5$ & $2.1 \pm 1.0 \ddagger$ & $3.0 \pm 0.4$ & $3.4 \pm 1.4$ & $4.3 \pm 1.2$ \\
\hline Mean ICP (mmHg) & $8.3 \pm 1.6$ & $6.1 \pm 3.4$ & $7.3 \pm 6.3$ & $6.4 \pm 8.3$ & $7.4 \pm 3.9$ & $8.7 \pm 2.3$ \\
\hline \multicolumn{7}{|l|}{ Plateau phase } \\
\hline MWA (mmHg) & $5.0 \pm 1.9 \dagger$ & $5.1 \pm 0.1 \dagger$ & $4.4 \pm 1.9 \S$ & $7.2 \pm 0.6$ & $9.0 \pm 3.4$ & $10.8 \pm 2.3$ \\
\hline Mean ICP (mmHg) & $17.1 \pm 3.9$ & $16.3 \pm 4.1$ & $19.5 \pm 6.6$ & $18.9 \pm 6.0$ & $23.1 \pm 6.8$ & $25.3 \pm 7.2$ \\
\hline \multicolumn{7}{|l|}{ Infusion phase } \\
\hline MWA (mmHg) & $4.1 \pm 1.3$ & $4.2 \pm 0.4$ & $3.5 \pm 1.4 \S$ & $5.5 \pm 0.3$ & $5.8 \pm 2.2$ & $7.4 \pm 1.5$ \\
\hline Mean ICP $(\mathrm{mmHg})$ & $13.4 \pm 2.5$ & $13.1 \pm 4.8$ & $14.8 \pm 6.3$ & $13.8 \pm 7.1$ & $15.5 \pm 4.0$ & $16.5 \pm 4.0$ \\
\hline ICC (ml/mmHg) & $0.60 \pm 0.7$ & $0.66 \pm 0.2$ & $0.64 \pm 0.11 \dagger$ & $0.78 \pm 0.22 \dagger$ & $0.52 \pm 0.12$ & $0.45 \pm 0.16$ \\
\hline $\mathrm{R}_{\text {out }}(\mathrm{mmHg} / \mathrm{ml} / \mathrm{min})$ & $5.9 \pm 2.8$ & $6.8 \pm 0.8$ & $8.1 \pm 2.1$ & $8.4 \pm 1.5$ & $10.5 \pm 4.4$ & $11.1 \pm 4.5$ \\
\hline Infusion volume (ml) & $20.1 \pm 4.6$ & $24.9 \pm 4.9 \ddagger$ & $18.5 \pm 3.7$ & $16.9 \pm 3.2$ & $15.1 \pm 5.5$ & $13.0 \pm 3.2$ \\
\hline
\end{tabular}

Data presented as mean \pm SD. Significant differences were determined relative to the surgery-responder $(\mathrm{cHC})$ subgroup using 1-way ANOVA and Bonferroni-corrected post hoc tests.

* Overnight ICP parameters were recorded from 11 PM to $7 \mathrm{AM}$.

$\dagger p<0.05$.

$\ddagger p<0.01$.

$\S p<0.001$

als requiring shunt surgery after ETV (ETV+shunt group). These observations illustrate that $\mathrm{ncHC}$ is a heterogeneous condition. A recent systematic review and meta-analysis concluded that there is not enough evidence to state whether ETV is superior to shunt placement in ncHC. ${ }^{38} \mathrm{In}$ the present cohort, a shunt after ETV was required in 21 $(38 \%)$ of 55 surgically treated individuals with ncHC. The proportion requiring a shunt following ETV varies greatly in the literature, ${ }^{38,44,45,47}$ thus the failure rate was $50 \%$ in 1 study ${ }^{45}$

\section{Monitoring of ICP and Ventricular Infusion Testing in Chronic NcHC}

In this institution, there is a long tradition of diagnostic overnight monitoring of $\mathrm{ICP}^{33,34}$ and ventricular infusion testing ${ }^{30,31}$ in patients with HC. Others also previously used diagnostic ventricular infusion testing in $\mathrm{HC} .{ }^{32,43}$ Based on monitoring of the static ICP (mean ICP), the $\mathrm{R}_{\text {out }}$ has been determined.

One interpretation of the present results of ventricular infusion testing is that it differentiates between impaired intraventricular CSF absorption and impaired extraventricular CSF absorption. According to this view, intact ventricular CSF absorption means that intraventricular CSF mixes freely with interstitial fluid (ISF) by free transependymal transport, while extraventricular CSF absorption means that CSF outside the ventricles mixes freely with subcortical ISF. This view is supported by the recently described concept of paravascular (glymphatic) transport within the human brain. ${ }^{27}$ The CSF/ISF may at least partly escape from the cranial cavity in lymphatic vessels located along venous vessels. ${ }^{29}$

\section{Pulsatile and Static ICP Scores in Chronic ncHC}

Overnight ICP monitoring and ventricular infusion testing revealed comparable pulsatile and static ICP scores in patients with chronic ncHC and the $\mathrm{cHC}$ cohort. Moreover, the ICP wave amplitudes were increased to a similar degree in patients with $\mathrm{ncHC}$ and the $\mathrm{cHC}$ patients who responded clinically to surgery. The levels of MWA in ncHC responders were comparable to that observed in other patient groups with CSF circulation failure and response to CSF diversion surgery, such as patients with idiopathic normal pressure hydrocephalus (iNPH), ${ }^{19}$ idiopathic intracranial hypertension (IIH) ${ }^{20}$ pediatric $\mathrm{HC},{ }^{42}$ and Chiari malformation Type $\mathrm{I}^{20}$ Also, patients 

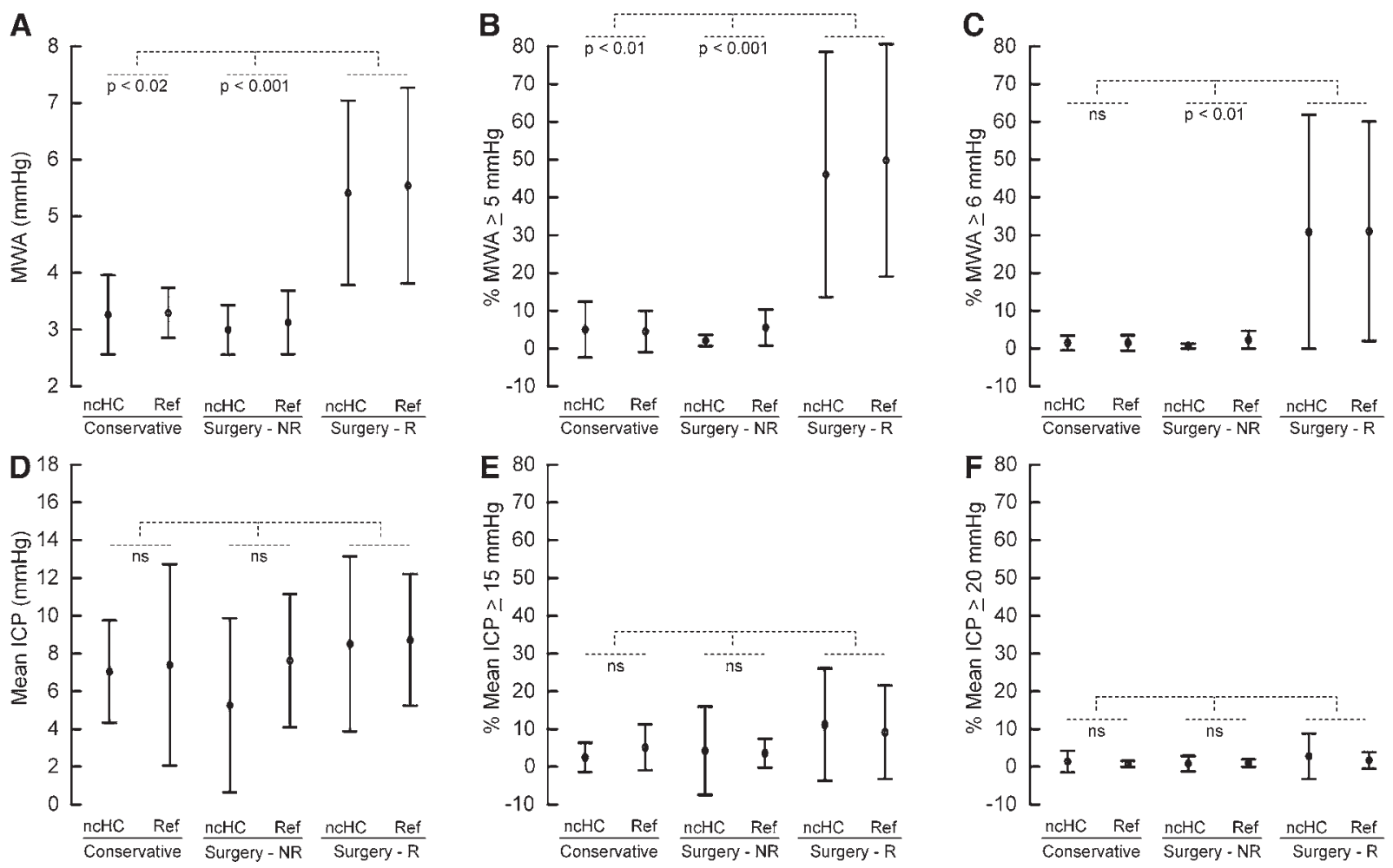

FIG. 3. Graphs of pulsatile ICP (MWA; A-C) and static ICP (mean ICP; D-F) scores during overnight monitoring from 11 PM to 7 AM for the 3 different management groups (conservative, surgery- $\mathrm{NR}$, and surgery- $\mathrm{R}$ ) of the 2 patient cohorts ( $\mathrm{ncHC}$ and $\mathrm{cHC}$ ). $\mathrm{MWA}$ was analyzed as the average MWA (A), percentage of MWA $\geq 5 \mathrm{~mm} \mathrm{Hg}(B)$, and percentage of MWA $\geq 6 \mathrm{~mm} \mathrm{Hg}(C)$. Mean ICP was analyzed as the mean ICP (D), percentage of mean ICP $\geq 15 \mathrm{~mm} \mathrm{Hg}(E)$, and percentage of mean ICP $\geq 20 \mathrm{~mm} H \mathrm{Hg}(\mathrm{F})$. Each error bar is presented as the mean \pm SD. Differences between the groups were determined by 1-way ANOVA and Bonferronicorrected post hoc tests. The main observation was that individuals responding to surgery (surgery-R) differed significantly from those not responding (surgery-NR) and those managed conservatively (conservative) for all MWA parameters $(A-C)$. Note that the MWA parameters were close to identical for the $\mathrm{ncHC}$ and reference $(\mathrm{cHC})$ cohorts. No significant differences were found for mean ICP. ns = nonsignificant.

with cerebral bleeds due to subarachnoid hemorrhage had MWA values in the same range. ${ }^{12}$ Based on measurements from several groups of patients with CSF circulation disorders in this institution, we consider an average MWA of $4 \mathrm{~mm} \mathrm{Hg}$ during overnight monitoring as the upper normal threshold, while an average MWA of 4-5 mm Hg represents borderline.

In patients with chronic ncHC (and $\mathrm{cHC}$ ), the static ICP (mean ICP) was modestly elevated with average values < $10 \mathrm{~mm} \mathrm{Hg}$ and a low percentage of ICP elevations $>15$ or $20 \mathrm{~mm} \mathrm{Hg}$. The static ICP values found in this cohort cannot be accompanied by impaired cerebral perfusion pressure, which requires higher static ICP. ${ }^{4}$ Even in patients responding to surgery, the static ICP was not significantly increased: neither the average of mean ICP or percentage $>15$ or $20 \mathrm{~mm} \mathrm{Hg}$ during overnight monitoring, nor the static ICP during ventricular infusion testing. Hence, the present data do not provide evidence of increased static ICP in chronic ncHC.

\section{ICC Scores in Chronic ncHC}

The present results of ventricular infusion testing provided evidence of impaired ICC in patients with ncHC responding to surgery. Moreover, in ncHC, there was a highly significant negative correlation between ICC and MWA during ventricular infusion testing and also between MWA measured overnight and ICC. These observations support the view that pulsatile ICP is indicative of ICC. The present data support results recently published by this author, both by measuring ICC with the Spiegelberg monitor ${ }^{17}$ and by measuring ICC during ventricular infusion testing ${ }^{11}$ in other patient groups.

ICC refers to the pressure-volume reserve capacity of the intracranial compartment. Impaired ICC means that a small intracranial volume change results in a disproportional pressure increase and elevated pulse pressure amplitudes. ${ }^{1,17}$

In the present patients with chronic ncHC, mean ICP was not significantly related to ICC. In addition, ICC was low $(<0.5 \mathrm{ml} / \mathrm{mmHg})$ despite mean ICP $<10-15 \mathrm{~mm} \mathrm{Hg}$, highlighting the observations that ICC may be reduced despite normal mean ICP. ${ }^{22}$

The present observations are supported by some previous invasive studies. In patients with ncHC due to aqueductal stenosis, Tisell et al. ${ }^{46}$ reported a significant positive correlation between outcome and preoperative intracranial elastance (ICE) measurement (i.e., ICE $=1 /$ ICC). In animals, measurements of ICC have shown a significant negative correlation between ICP wave amplitudes. ${ }^{23}$ Furthermore, animal experiments showed that shunt implan- 
TABLE 4. The ICP/infusion test scores of ncHC patients categorized according to mode of surgery

\begin{tabular}{|c|c|c|c|}
\hline \multirow[b]{2}{*}{ Testing Variable } & \multirow{2}{*}{$\begin{array}{l}\text { Surgery Group } \\
\text { (nonresponders) }\end{array}$} & \multicolumn{2}{|c|}{ Surgery Group (responders) } \\
\hline & & ETV & ETV-Shunt \\
\hline \multicolumn{4}{|l|}{ Overnight ICP monitoring* } \\
\hline \multicolumn{4}{|l|}{ MWA } \\
\hline Average $(\mathrm{mmHg})$ & $3.0 \pm 0.4$ & $5.2 \pm 1.7 \S$ & $5.7 \pm 1.6 \S$ \\
\hline$\% \geq 5 \mathrm{mmHg}$ & $2 \pm 2$ & $42 \pm 32 \ddagger$ & $53 \pm 34 \S$ \\
\hline$\% \geq 6 \mathrm{mmHg}$ & $0 \pm 1$ & $27 \pm 30 \dagger$ & $36 \pm 34 \ddagger$ \\
\hline \multicolumn{4}{|l|}{ Mean ICP } \\
\hline Average $(\mathrm{mmHg})$ & $5.2 \pm 4.6$ & $7.9 \pm 3.6$ & $9.3 \pm 5.9$ \\
\hline$\% \geq 15 \mathrm{mmHg}$ & $4 \pm 12$ & $8 \pm 11$ & $15 \pm 19$ \\
\hline$\% \geq 20 \mathrm{mmHg}$ & $1 \pm 2$ & $2 \pm 2$ & $4 \pm 9$ \\
\hline \multicolumn{4}{|l|}{ Ventricular infusion testing } \\
\hline \multicolumn{4}{|l|}{ Opening phase } \\
\hline MWA (mmHg) & $2.1 \pm 1.0$ & $3.3 \pm 1.6$ & $3.4 \pm 1.2$ \\
\hline Mean ICP (mmHg) & $7.3 \pm 6.3$ & $8.0 \pm 4.4$ & $6.4 \pm 3.0$ \\
\hline \multicolumn{4}{|l|}{ Plateau phase } \\
\hline MWA (mmHg) & $4.4 \pm 1.9$ & $8.7 \pm 3.5 \ddagger$ & $9.3 \pm 3.3 \S$ \\
\hline Mean ICP (mmHg) & $19.5 \pm 6.6$ & $23.4 \pm 6.1$ & $22.7 \pm 8.0$ \\
\hline \multicolumn{4}{|l|}{ Infusion phase } \\
\hline MWA (mmHg) & $3.5 \pm 1.4$ & $5.7 \pm 2.2 \dagger$ & $6.1 \pm 2.2 \ddagger$ \\
\hline Mean ICP $(\mathrm{mmHg})$ & $14.8 \pm 6.4$ & $16.0 \pm 4.3$ & $14.9 \pm 3.6$ \\
\hline $\mathrm{ICC}(\mathrm{ml} / \mathrm{mmHg})$ & $0.64 \pm 0.11$ & $0.51 \pm 0.12 \dagger$ & $0.52 \pm 0.11$ \\
\hline $\mathrm{R}_{\text {out }}(\mathrm{mmHg} / \mathrm{ml} / \mathrm{min})$ & $8.1 \pm 2.1$ & $10.3 \pm 4.5$ & $10.9 \pm 4.5$ \\
\hline Infusion volume (ml) & $18.5 \pm 3.7$ & $15.4 \pm 6.3$ & $14.8 \pm 4.3$ \\
\hline \multicolumn{4}{|c|}{$\begin{array}{l}\text { Data presented as mean } \pm \text { SD. Significant differences between nonresponders and responders were determined by 1-way ANOVA with } \\
\text { Bonferroni-corrected post hoc test. } \\
\text { * Overnight ICP parameters were recorded from } 11 \text { PM to } 7 \mathrm{AM} . \\
\dagger p<0.05 . \\
\begin{array}{l}f \\
\oint\end{array}<0.01 . \\
\S p<0.001 .\end{array}$} \\
\hline
\end{tabular}

tation may alter ICC, as well as brain tissue oxygenation in chronic obstructive $\mathrm{HC}$, despite the mean ICP being within the normal range. ${ }^{22}$

\section{Impaired ICC and Chronic HC}

The present findings of increased pulsatile ICP and reduced ICC in chronic ncHC, despite normal ICP, may explain why these patients with normal ICP respond to CSF diversion procedures. We have previously suggested that impaired ICC, despite normal ICP, may be a common mechanism behind different conditions characterized by abnormal CSF circulation, including diseases such as iNPH ${ }^{19}{ }^{1 I H},{ }^{20}$ pediatric $\mathrm{HC},{ }^{42}$ and Chiari malformation. ${ }^{20}$ The rationale behind the hypothesis is findings of increased pulsatile ICP in patients responding clinically to CSF diversion procedures. The present observations support the concept of impaired ICC in treatment-dependent ncHC. ${ }^{24}$

While the present results were based on invasive measurements, several other authors have argued in favor of impaired ICC in chronic HC based on findings from MRI. Using phase-contrast MRI, Bateman found evidence of reduced venous compliance and increased venous pressure in late-onset aqueductal stenosis. ${ }^{2}$ Furthermore, from MRI measurements Greitz hypothesized that impaired ICC is a common mechanism of all chronic HC types. ${ }^{24,25} \mathrm{Im}$ paired ICC was also the proposed mechanism underlying the clinical entities LIAS $^{21}$ and LOVA. ${ }^{36}$

Independent of $\mathrm{HC}$ type and treatment modality, the goal of treatment is to prevent brain damage by maintaining adequate cerebral blood perfusion and tissue oxygenation. ${ }^{22}$ Provided impaired ICC is a key feature behind chronic HC, the goal of treatment is to improve ICC. The present observations support this view. In general, shunting may have a greater overall effect on ICC, blood volume, and oxygenation than ETV. ${ }^{24,25}$ Shunting reduces the intracranial CSF volume, which may have a direct effect on venous dilation. On the other hand, ETV has an indirect effect by facilitating the ventricular CSF outflow and thereby slightly reducing the parenchymal systolic pressure. Reduced systolic forces reduce the compression of intracranial veins and capillaries but with lesser effect on the venous compliance than the effects observed in shunt placement.

The present results of ventricular infusion testing suggest that deficient ventricular CSF absorption is one mechanism in those responding to ETV, while deficient intraventricular and extraventricular CSF absorption is involved in those requiring both ETV and a shunt. Ven- 

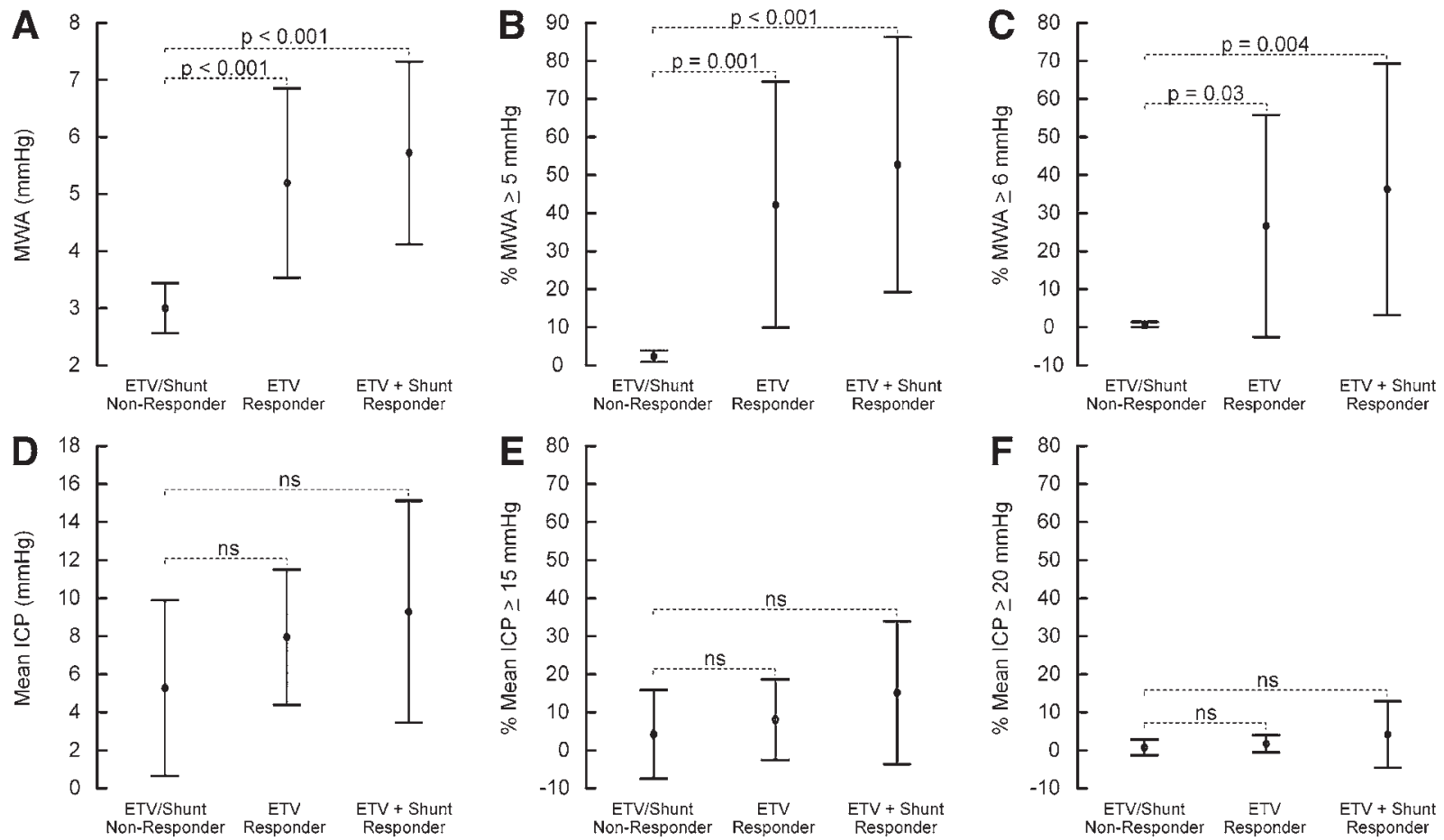

FIG. 4. Graphs of pulsatile ICP (MWA, A-C) and static ICP (mean ICP, D-F) scores during overnight monitoring from 11 PM to 7 AM for the $\mathrm{ncHC}$ patients treated surgically (ETV or ETV+shunt), and categorized according to clinical response (nonresponder or responder). MWA was analyzed as the average MWA (A), percentage of MWA $\geq 5 \mathrm{~mm} \mathrm{Hg}(B)$, and percentage of $M W A \geq 6 \mathrm{~mm}$ $\mathrm{Hg}(C)$. Mean ICP was analyzed as the mean ICP (D), percentage of mean ICP $\geq 15 \mathrm{~mm} \mathrm{Hg}(\mathrm{E})$, and percentage of mean ICP $\geq$ $20 \mathrm{~mm} \mathrm{Hg}(\mathrm{F})$. Each error bar is presented as the mean \pm SD. Differences between groups were determined by 1-way ANOVA and Bonferroni-corrected post hoc tests. The main observation was that patients responding to surgery (both ETV responders and ETV+shunt responders) differed significantly from those not responding (ETV+shunt nonresponders) for all MWA parameters $(A-C)$. Note that the MWA parameters were close to identical for the ETV responders and ETV+shunt responders. No significant differences were observed for mean ICP. ns = nonsignificant.

tricular CSF absorption may play an important role in some kinds of HC. Hence, in iNPH, phase-contrast MRI revealed net retrograde aqueductal CSF flow, i.e., net flow toward the ventricle, which was reversed after shunting. ${ }^{40}$ These results provided evidence that in iNPH, intraven- tricular CSF absorption was increased while extraventricular (cortical) absorption reduced.

Several lines of evidence suggest that vascular disease is linked to the development of chronic HC. ${ }^{9}$ We recently reported increased prevalence of cardiovascular disease in
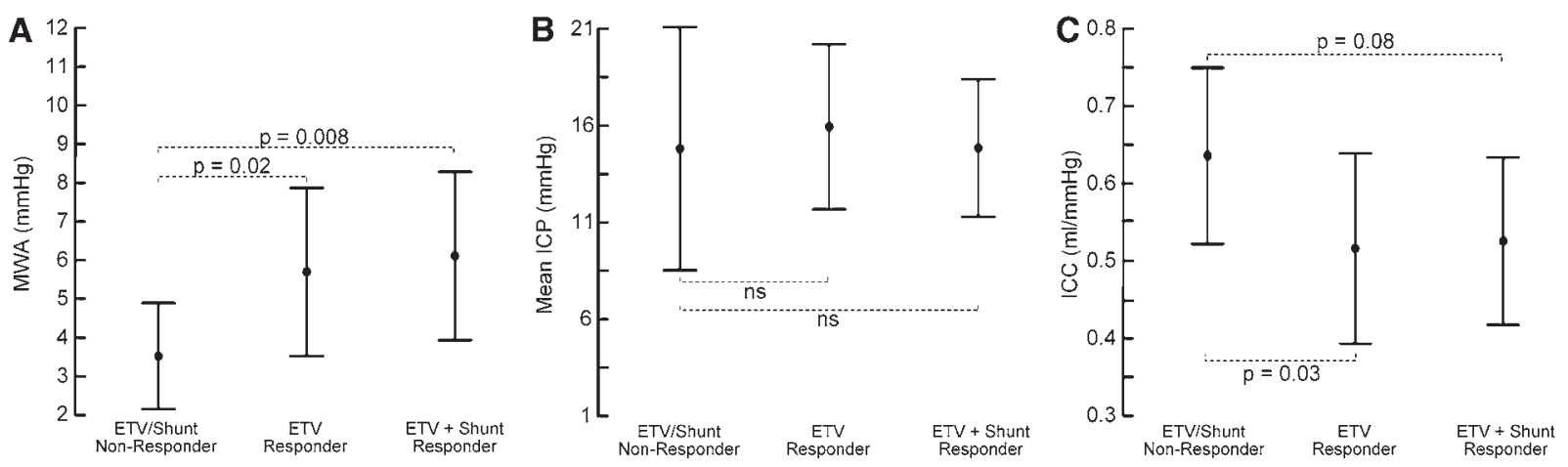

FIG. 5. The pulsatile ICP (MWA; A), static ICP (mean ICP; B), and ICC (C) scores during the infusion phase of ventricular infusion testing are shown for the patients with $\mathrm{ncHC}$ who were treated surgically (ETV or ETV+shunt), and categorized according to clinical response (nonresponder or responder). During the infusion phase of ventricular infusion testing, the average MWA ( $A$ ), mean $I C P(B)$, and ICC $(C)$ were compared between the different management groups. Each error bar is presented as the mean $\pm S D$. Differences between groups were determined by 1-way ANOVA and Bonferroni-corrected post hoc tests. The main observation was that individuals responding to surgery (both ETV responders and ETV+shunt responders) had significantly higher MWA (A) and lower ICC (C) than those not responding (ETV+shunt nonresponders). No significant differences were observed for mean ICP. ns = nonsignificant. 

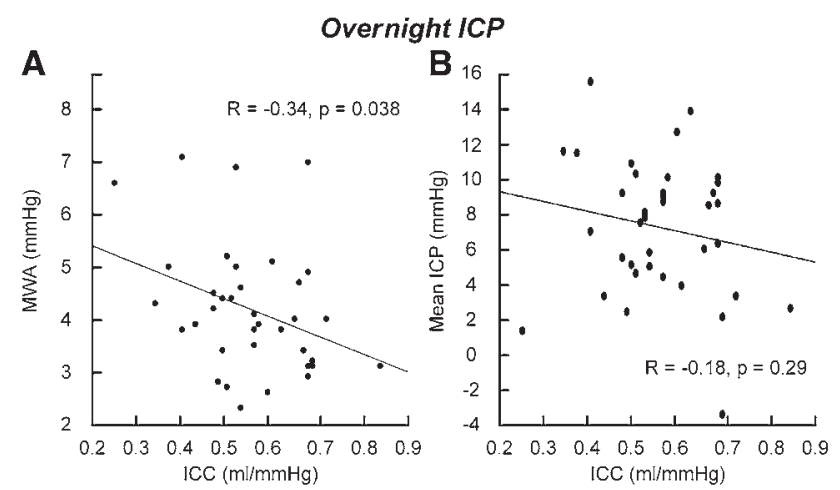

FIG. 6. Graphs of the association between ICC determined during ventricular infusion testing and average MWA $(\mathbf{A})$ and mean ICP $(\mathbf{B})$ determined during overnight monitoring from $11 \mathrm{PM}$ to $7 \mathrm{AM}$. The fit lines with Pearson correlation coefficients $(R)$ and significance levels show a significant negative correlation between MWA and ICC, but not between mean ICP and ICC. From the ventricular infusion testing is presented the association between ICC and MWA (C) and mean ICP (D) during the opening phase, between ICC and MWA (E) and mean ICP $(F)$ during the plateau phase, and between ICC and MWA $(\mathbf{G})$ and mean ICP $(\mathbf{H})$ during the infusion phase. The fit lines with the Pearson correlation coefficients and significance levels show a significant negative correlation between ICC and MWA during the opening $(C)$, plateau $(E)$, and infusion $(G)$ phases of the infusion test, but no significant correlations between ICC and mean ICP.

chronic ncHC due to aqueductal stenosis, ${ }^{16}$ and in iNPH. ${ }^{15}$ Pathological changes in the ependymal cells have been shown in HC, which may be a cause of reduced transepen-
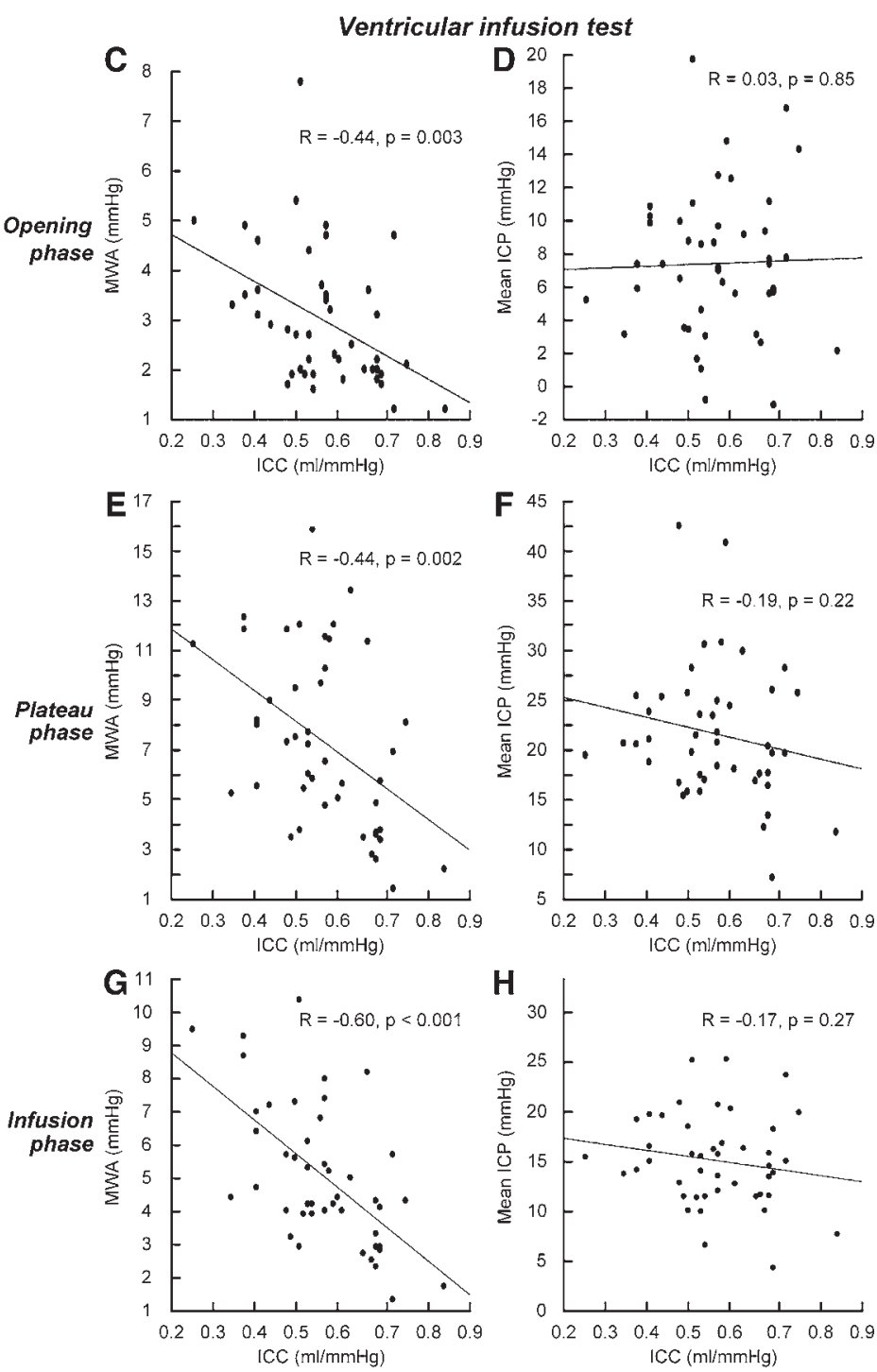

dymal water flux in some patients. ${ }^{6-8}$ However, this study presented a few individuals with chronic ncHC due to aqueductal stenosis who managed well without surgery and

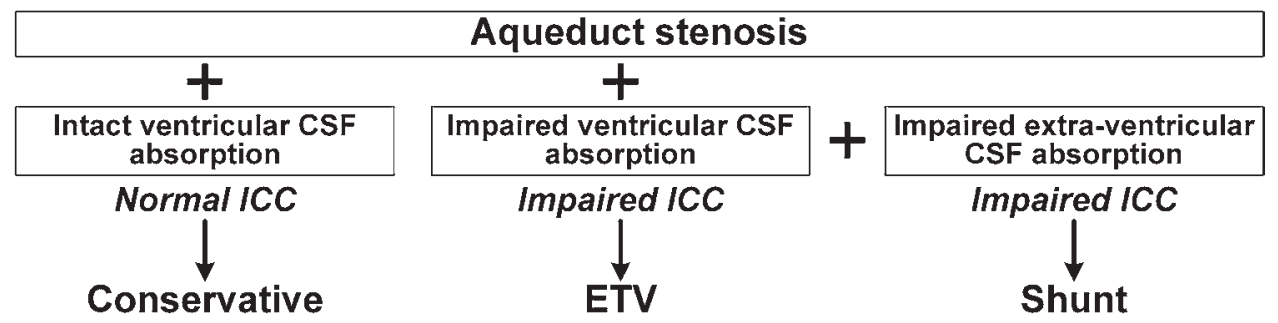

FIG. 7. Summary of the possible pathophysiological mechanisms behind $\mathrm{ncHC}$ caused by aqueductal stenosis, which are supported by the present results of overnight ICP monitoring and ventricular infusion testing. In patients with aqueductal stenosis, a major issue is whether ventricular CSF absorption is intact (the transependymal CSF flow is normal) or impaired (transependymal CSF flow is hampered). In the latter case, ICC (pressure-volume reserve capacity) may become reduced and be accompanied by symptom development. In this situation, ETV may be successful by establishing an alternative route for CSF flow. However, a requirement for the success of ETV is that extraventricular CSF absorption is intact. When both intraventricular and extraventricular CSF absorption is impaired, CSF diversion by shunt surgery may be required to counteract the impaired compliance. The present results do not provide evidence about the exact site for CSF absorption, and exactly how impaired CSF absorption leads to impaired ICC. Further studies, including MRI modalities, may be useful to clarify this issue. 
in whom overnight ICP monitoring and ventricular infusion testing revealed normal findings. In these patients, it is argued that ventricular CSF absorption is normal, also keeping ICC normal. This observation may explain why some patients with ncHC due to aqueductal stenosis manage well without surgery.

Based on the present observations, it is suggested that patients with impaired ventricular CSF absorption are those with ncHC who are at risk. If fluid cannot escape from the ventricles due to CSF obstruction and obstruction of transependymal transport, ICC is becoming reduced and energy supply to the brain is hampered. This tentative view is illustrated in Fig. 7. Further studies, using MRI modalities, are in progress to study this issue.

\section{Conclusions}

The present study provides new insight into the pathophysiology of chronic ncHC. First, impaired ICC characterizes patients with chronic ncHC who respond clinically to surgery, while increased static ICP appears to play a less significant role. Second, in patients responding clinically to ETV, impaired ventricular CSF absorption is a key player. Patients requiring shunt placement for clinical response may have both intraventricular and extraventricular CSF absorption failure. A subgroup of patients with ncHC due to aqueductal stenosis may have normal ventricular CSF absorption and normal ICC and may not require surgery.

\section{References}

1. Avezaat CJ, van Eijndhoven JH, Wyper DJ: Cerebrospinal fluid pulse pressure and intracranial volume-pressure relationships. J Neurol Neurosurg Psychiatry 42:687-700, 1979

2. Bateman GA: Magnetic resonance imaging quantification of compliance and collateral flow in late-onset idiopathic aqueductal stenosis: venous pathophysiology revisited. J Neurosurg 107:951-958, 2007

3. Cinalli G, Spennato P, Ruggiero C, Aliberti F, Zerah M, Trischitta V, et al: Intracranial pressure monitoring and lumbar puncture after endoscopic third ventriculostomy in children. Neurosurgery 58:126-136, 2006

4. Czosnyka M, Pickard JD: Monitoring and interpretation of intracranial pressure. J Neurol Neurosurg Psychiatry 75:813-821, 2004

5. Dandy WE, Blackfan KD: Internal hydrocephalus: An experimental, clinical and pathological study. Am J Dis Child 8:406-482, 1914

6. Del Bigio MR: Ependymal cells: biology and pathology. Acta Neuropathol 119:55-73, 2010

7. Del Bigio MR: Neuropathology and structural changes in hydrocephalus. Dev Disabil Res Rev 16:16-22, 2010

8. Del Bigio MR, Bruni JE: Periventricular pathology in hydrocephalic rabbits before and after shunting. Acta Neuropathol 77:186-195, 1988

9. Edwards RJ, Dombrowski SM, Luciano MG, Pople IK: Chronic hydrocephalus in adults. Brain Pathol 14:325-336, 2004

10. Eide PK: The correlation between pulsatile intracranial pressure and indices of intracranial pressure-volume reserve capacity: results from ventricular infusion testing. J Neurosurg 125:1493-1503, 2016

11. Eide PK: A new method for processing of continuous intracranial pressure signals. Med Eng Phys 28:579-587, 2006
12. Eide PK, Bentsen G, Sorteberg AG, Marthinsen PB, Stubhaug A, Sorteberg W: A randomized and blinded singlecenter trial comparing the effect of intracranial pressure and intracranial pressure wave amplitude-guided intensive care management on early clinical state and 12-month outcome in patients with aneurysmal subarachnoid hemorrhage. Neurosurgery 69:1105-1115, 2011

13. Eide PK, Brean A: Cerebrospinal fluid pulse pressure amplitude during lumbar infusion in idiopathic normal pressure hydrocephalus can predict response to shunting. Cerebrospinal Fluid Res 7:5, 2010

14. Eide PK, Lundar T: Arne Torkildsen and the ventriculocisternal shunt: the first clinically successful shunt for hydrocephalus. J Neurosurg 124:1421-1428, 2016

15. Eide PK, Pripp AH: Increased prevalence of cardiovascular disease in idiopathic normal pressure hydrocephalus patients compared to a population-based cohort from the HUNT3 survey. Fluids Barriers CNS 11:19, 2014

16. Eide PK, Pripp AH: The prevalence of cardiovascular disease in non-communicating hydrocephalus. Clin Neurol Neurosurg 149:33-38, 2016

17. Eide PK, Sorteberg W: Association among intracranial compliance, intracranial pulse pressure amplitude and intracranial pressure in patients with intracranial bleeds. Neurol Res 29:798-802, 2007

18. Eide PK, Sorteberg W: Diagnostic intracranial pressure monitoring and surgical management in idiopathic normal pressure hydrocephalus: a 6-year review of 214 patients. Neurosurgery 66:80-91, 2010

19. Eide PK, Sorteberg W: Outcome of surgery for idiopathic normal pressure hydrocephalus: role of preoperative static and pulsatile intracranial pressure. World Neurosurg 86:186-193, 193.e1, 2016

20. Frič R, Eide PK: Comparative observational study on the clinical presentation, intracranial volume measurements, and intracranial pressure scores in patients with either Chiari malformation Type I or idiopathic intracranial hypertension. J Neurosurg 126:1312-1322, 2017

21. Fukuhara T, Luciano MG: Clinical features of late-onset idiopathic aqueductal stenosis. Surg Neurol 55:132-136, discussion 136-137, 2001

22. Fukuhara T, Luciano MG, Brant CL, Klauscie J: Effects of ventriculoperitoneal shunt removal on cerebral oxygenation and brain compliance in chronic obstructive hydrocephalus. J Neurosurg 94:573-581, 2001

23. González-Darder JM, Barcia-Salorio JL: Pulse amplitude and volume-pressure relationships in experimental hydrocephalus. Acta Neurochir (Wien) 97:166-170, 1989

24. Greitz D: The hydrodynamic hypothesis versus the bulk flow hypothesis. Neurosurg Rev 27:299-300, 2004

25. Greitz D: Paradigm shift in hydrocephalus research in legacy of Dandy's pioneering work: rationale for third ventriculostomy in communicating hydrocephalus. Childs Nerv Syst 23:487-489, 2007

26. Harrison MJ, Robert CM, Uttley D: Benign aqueduct stenosis in adults. J Neurol Neurosurg Psychiatry 37:1322-1328, 1974

27. Iliff JJ, Wang M, Liao Y, Plogg BA, Peng W, Gundersen GA, et al: A paravascular pathway facilitates CSF flow through the brain parenchyma and the clearance of interstitial solutes, including amyloid $\beta$. Sci Transl Med 4:147ra111, 2012

28. Kulkarni AV, Drake JM, Kestle JR, Mallucci CL, Sgouros S, Constantini S: Endoscopic third ventriculostomy vs cerebrospinal fluid shunt in the treatment of hydrocephalus in children: a propensity score-adjusted analysis. Neurosurgery 67:588-593, 2010

29. Louveau A, Smirnov I, Keyes TJ, Eccles JD, Rouhani SJ, Peske JD, et al: Structural and functional features of central nervous system lymphatic vessels. Nature 523:337-341, 2015 
30. Lundar T, Nornes H: Determination of ventricular fluid outflow resistance in patients with ventriculomegaly. J Neurol Neurosurg Psychiatry 53:896-898, 1990

31. Magnaes B: Hydromechanical testing in non-communicating hydrocephalus to select patients for microsurgical third ventriculostomy. Br J Neurosurg 3:443-450, 1989

32. Morgan MK, Johnston IH, Spittaler PJ: A ventricular infusion technique for the evaluation of treated and untreated hydrocephalus. Neurosurgery 29:832-837, 1991

33. Nornes H, Aaslid R, Lindegaard KF: Intracranial pulse pressure dynamics in patients with intracranial hypertension. Acta Neurochir (Wien) 38:177-186, 1977

34. Nornes H, Serck-Hanssen F: Miniature transducer for intracranial pressure monitoring in man. Acta Neurol Scand 46:203-214, 1970

35. Oi S: Classification of hydrocephalus: critical analysis of classification categories and advantages of "Multi-categorical Hydrocephalus Classification" (Mc HC). Childs Nerv Syst 27:1523-1533, 2011

36. Oi S, Shimoda M, Shibata M, Honda Y, Togo K, Shinoda M, et al: Pathophysiology of long-standing overt ventriculomegaly in adults. J Neurosurg 92:933-940, 2000

37. Rapanà $\mathrm{A}$, Bellotti $\mathrm{A}$, Iaccarino $\mathrm{C}$, Pascale $\mathrm{M}$, Schönauer $\mathrm{M}$ : Intracranial pressure patterns after endoscopic third ventriculostomy. Preliminary experience. Acta Neurochir (Wien) 146:1309-1315, 2004

38. Rasul FT, Marcus HJ, Toma AK, Thorne L, Watkins LD: Is endoscopic third ventriculostomy superior to shunts in patients with non-communicating hydrocephalus? A systematic review and meta-analysis of the evidence. Acta Neurochir (Wien) 155:883-889, 2013

39. Rekate HL: A consensus on the classification of hydrocephalus: its utility in the assessment of abnormalities of cerebrospinal fluid dynamics. Childs Nerv Syst 27:1535-1541, 2011

40. Ringstad G, Emblem KE, Eide PK: Phase-contrast magnetic resonance imaging reveals net retrograde aqueductal flow in idiopathic normal pressure hydrocephalus. J Neurosurg 124:1850-1857, 2016

41. Sæhle T, Eide PK: Association between ventricular volume measures and pulsatile and static intracranial pressure scores in non-communicating hydrocephalus. J Neurol Sci 350:3339,2015

42. Sæhle T, Eide PK: Characteristics of intracranial pressure (ICP) waves and ICP in children with treatment-responsive hydrocephalus. Acta Neurochir (Wien) 157:1003-1014, 2015

43. Tans JT, Poortvliet DC: Comparison of ventricular steadystate infusion with bolus infusion and pressure recording for differentiating between arrested and non-arrested hydrocephalus. Acta Neurochir (Wien) 72:15-29, 1984

44. Tisell M: How should primary aqueductal stenosis in adults be treated? A review. Acta Neurol Scand 111:145-153, 2005

45. Tisell M, Almström O, Stephensen H, Tullberg M, Wikkelsö C: How effective is endoscopic third ventriculostomy in treating adult hydrocephalus caused by primary aqueductal stenosis? Neurosurgery 46:104-111, 2000

46. Tisell M, Edsbagge M, Stephensen H, Czosnyka M, Wikkelsø $\mathrm{C}$ : Elastance correlates with outcome after endoscopic third ventriculostomy in adults with hydrocephalus caused by primary aqueductal stenosis. Neurosurgery 50:70-77, 2002

47. Tisell M, Hellström P, Ahl-Börjesson G, Barrows G, Blomsterwall E, Tullberg M, et al: Long-term outcome in 109 adult patients operated on for hydrocephalus. Br J Neurosurg 20:214-221, 2006

48. Vogel TW, Bahuleyan B, Robinson S, Cohen AR: The role of endoscopic third ventriculostomy in the treatment of hydrocephalus. J Neurosurg Pediatr 12:54-61, 2013

\section{Disclosures}

Dr. Eide has a financial interest in the software company (dPCom AS, Oslo) manufacturing the software (Sensometrics Software) used for analysis of the ICP recordings.

\section{Correspondence}

Per Kristian Eide, Department of Neurosurgery, Oslo University Hospital-Rikshospitalet, PB 4950 Nydalen, Oslo 0424, Norway. email: peide@ous-hf.no. 